\title{
Near-Edge Soft X-ray Absorption Mass Spectrometry of Protonated Melittin
}

\author{
Dmitrii Egorov, ${ }^{1}$ Sadia Bari, ${ }^{2}$ Rebecca Boll, ${ }^{2}$ Simon Dörner, ${ }^{2}$ Sascha Deinert, ${ }^{2}$ \\ Simone Techert, ${ }^{2,3}$ Ronnie Hoekstra, ${ }^{1}$ Vicente Zamudio-Bayer, ${ }^{4,5,6}$ Rebecka Lindblad, ${ }^{5,7}$ \\ Christine Bülow, ${ }^{4,5,8}$ Martin Timm, ${ }^{4,5,8}$ Bernd von Issendorff, ${ }^{6}$ J. Tobias Lau, ${ }^{4,5}$ \\ Thomas Schlathölter ${ }^{1}$ (
}

${ }^{1}$ Zernike Institute for Advanced Materials, University of Groningen, Nijenborgh 4, 9747AG, Groningen, Netherlands

${ }^{2}$ Deutsches Elektronen-Synchrotron DESY, Notkestr. 85, 22607, Hamburg, Germany

${ }^{3}$ Institute of X-ray Physics, University of Göttingen, Friedrich-Hund-Platz 1, 37077, Göttingen, Germany

${ }^{4}$ Institut für Methoden und Instrumentierung der Forschung mit Synchrotronstrahlung, Helmholtz-Zentrum Berlin für Materialien und Energie, Albert-Einstein-Str. 15, 12489, Berlin, Germany

${ }^{5}$ Abteilung für Hochempfindliche Röntgenspektroskopie, Helmholtz-Zentrum Berlin für Materialien und Energie, Albert-EinsteinStr. 15, 12489, Berlin, Germany

${ }^{6}$ Physikalisches Institut, Universität Freiburg, Hermann-Herder-Str. 3, 79104, Freiburg, Germany

${ }^{7}$ Synkrotronljusfysik, Lunds Universitet, 22100, Lund, Sweden

${ }^{8}$ Institut für Optik und Atomare Physik, Technische Universität Berlin, Hardenbergstr. 36, 10623, Berlin, Germany
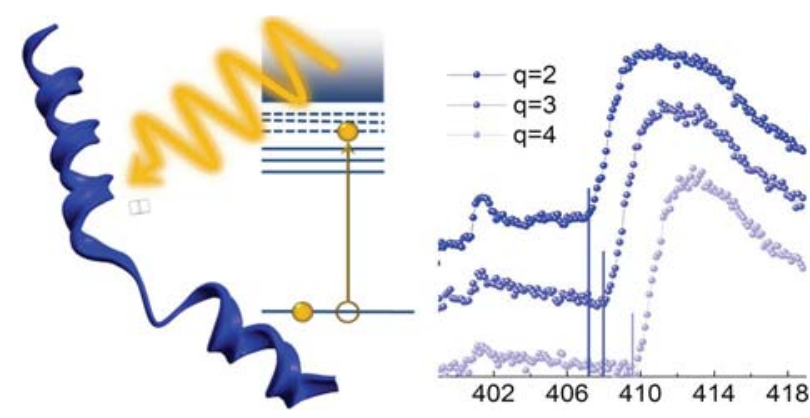

Abstract. We have investigated the photoionization and photofragmentation yields of gas-phase multiply protonated melittin cations for photon energies at the K-shell absorption edges of carbon, nitrogen, and oxygen. Two similar experimental approaches were employed. In both experiments, mass selected [melittin+qH] ${ }^{q+}(q=2-4)$ ions were accumulated in radiofrequency ion traps. The trap content was exposed to intense beams of monochromatic soft X-ray photons from synchrotron beamlines and photoproducts were analyzed by means of time-of-flight mass spectrometry. Mass spectra were recorded for fixed photon energies, and partial ion yield spectra were recorded as a function of photon energy. The combination of mass spectrometry and soft X-ray spectroscopy allows for a direct correlation of protein electronic structure with various photoionization channels. Non-dissociative single and double ionization are used as a reference. The contribution of both channels to various backbone scission channels is quantified and related to activation energies and protonation sites. Soft X-ray absorption mass spectrometry combines fast energy deposition with single and double ionization and could complement established activation techniques.

Keywords: Soft X-ray spectroscopy, Soft X-ray absorption, Photodissociation, Melittin, Protonated proteins

Received: 28 May 2018/Revised: 10 July 2018/Accepted: 10 July 2018/Published Online: 25 July 2018 Electronic supplementary material The online version of this article
(https://doi.org/10.1007/s13361-018-2035-6) contains supplementary material, which is available to authorized users.

Correspondence to: Thomas Schlathölter; e-mail: t.a.schlatholter@rug.nl

\section{Introduction}

$\mathrm{T}$ of analysis of the molecular fragmentation pattern. Established activation methods can be classified as either collisional, 
electronic, or photonic. In collision-based methods, excitation of the molecules is achieved in collisions with atoms (collisioninduced dissociation, CID [1]), surfaces (surface-induced dissociation, SID [2]), or ions [3-5]. Electron-based methods employ the attachment of electrons (e.g., electron captureinduced dissociation, ECD [6]) or on their detachment (e.g., charge transfer dissociation, CTD [7]). The third class of activation methods is photon-based and includes established techniques such as infrared multiphoton dissociation (IRMPD [8]) or ultraviolet photodissociation (UVPD [9]). Over the last few years, the potential of more energetic vacuum ultraviolet photons for mass spectrometry was explored, by interfacing tandem mass spectrometers with synchrotron beamlines. In pioneering experiments, for the protonated peptide leucine enkephalin, the resulting photofragmentation spectra were found to be dominated by immonium ions and small sequence ions [10], whereas for the much larger multiply protonated protein cytochrome $\mathrm{c}$, non-dissociative photoionization was found to dominate [11].

It was a straightforward next step to increase the photon energy into the soft X-ray regime. Soft X-ray spectroscopy is based on excitation or ionization of inner-shell electrons and therefore element specific and sensitive to the local electronic structure. For amino acids, peptides, and proteins, soft Xray spectroscopy has mostly been performed using thin films [12-14]. Soft X-ray spectra obtained for different proteins were found to share a common structure with some characteristic differences, most of which are indicative of the particular amino acid composition of the protein [14].

First gas-phase experiments on the $\mathrm{C} \mathrm{K}$-edge of protonated leucine enkephalin [15] and on the C, N, and O K-edges of multiply protonated cytochrome $\mathrm{c}$ both revealed similar spectral features as observed in the condensed phase, such as for instance the main resonance being due to $1 \mathrm{~s}-\pi^{*}$ excitations in the amide $\mathrm{C}=\mathrm{O}$ bonds. However, the mass spectrometric approach adds the dimension of fragmentation channel to the soft $\mathrm{X}$-ray spectroscopic data. For leucine enkephalin, it was for example shown that soft X-ray absorption in the sidechains of aromatic amino acids leads to formation of fewer small immonium ion fragments than does absorption in the amide groups [15]. Milosavljević and co-workers found that 1s ionization energies of multiply protonated ubiquitin do not systematically increase with protein protonation state. Instead, over a wide range of protonation states, protein unfolding due to Coulomb repulsion was found to compensate the increase in protonation state, resulting in a near-constant $1 \mathrm{~s}$ ionization energy [16]. Very recently, in two complementary studies, the interplay between protein size and photoexcitation energy was investigated. Egorov et al. $[17,18]$ systematically investigated soft X-ray- and EUV-induced protein fragmentation in the $0.5-12-\mathrm{kDa}$ range and found fragmentation yields and patterns to largely scale with protein temperature with large proteins being mainly subject to non-dissociative ionization. On the other hand, when multiple $90-\mathrm{eV}$ photons are absorbed simultaneously by a large protonated protein in a free-electron laser pulse, localized and non-ergodic fragmentation channels seem to dominate and the system behaves like an ensemble of small isolated peptides/amino acids [19].

The focus of this article is an in-depth X-ray spectrometry study on the small multiply protonated protein melittin. Melittin has an average mass of 2846.5 Da and with this intermediate size, it is expected to show a very rich fragmentation pattern, featuring immonium ions, sequence ions, and non-dissociative ionization.

For protonation states 2 and 3 , melittin is known to exhibit a primarily helical gas-phase secondary structure, which is lost upon increasing protonation to 4 [20]. This renders melittin an ideal system to study the interplay of structure and fragmentation upon soft X-ray absorption. In a previous work on soft Xray spectroscopy of cryogenic $(T \sim 10 \mathrm{~K})$ multiply protonated melittin at the $\mathrm{C} \mathrm{K}$-edge, we have recently found a correlation between melittin structure and electron impact ionization from within [21], i.e., inelastic scattering of photoelectrons along their trajectories through the photoabsorbing molecule. For low protonation, i.e., a compact structure, resonant $1 \mathrm{~s}-\pi_{\mathrm{C}=\mathrm{O}}^{*}$ excitation in an amide bond is significantly more likely to be followed by two-electron emission than for high protonation and elongated conformations. This is due to the differences in the integral cross section for electron impact ionization "from within," i.e., ionization by the Auger electron emitted after photoexcitation. Here, we will further investigate this effect for protonated melittin close to room temperature and at all three relevant K-edges of carbon, nitrogen, and oxygen. Furthermore, it is the goal of the article to investigate soft X-rayinduced protein fragmentation in greater detail in order to assess the potential of the technique for analytical mass spectrometry.

\section{Experimental}

All experiments were performed at the BESSY II synchrotron (Helmholtz-Zentrum Berlin für Materialien und Energie, Germany). Two similar experimental setups were used, both of which are based on the same concept: The gas-phase protonated protein of interest is first mass selected and subsequently trapped in a radiofrequency (RF) ion trap. The trapped ions are then exposed to a beam of soft X-ray photons. Eventually, the photoproducts are extracted into a time-of-flight mass spectrometer. The details, however, are different, allowing for complementary experiments.

\section{The Groningen Tandem Mass Spectrometer}

This apparatus has been described in detail before [10, 18]. Briefly, the protonated peptides are brought into the gas-phase by means of a home-built high-fluence electrospray ionization (ESI) source, phase space compressed by an RF ion funnel, collected in an RF octopole ion trap/guide, mass selected by an $\mathrm{RF}$ quadrupole mass filter, and collected in a 3D ion trap, of classical Paul trap geometry (Jordan TOF Products, CA). The ions are pre-trapped in the octopole for several $100 \mathrm{~ms}$ and 
bunched into the ion trap during less than $50 \mathrm{~ms}$. He buffer gas at $T=300 \mathrm{~K}$ is injected into the Paul trap only during the 50-ms filling period. For the experiments presented here, the Paul trap was interfaced with the beamline to expose the trap content to monochromatic soft X-ray photons at the C K-edge $(E=280-$ $300 \mathrm{eV}, 350 \mathrm{meV}$ bandwidth, typically $2 \times 10^{13}$ photons/s). Typical photoexposure times of several $100 \mathrm{~ms}$ lead to photoabsorption in $5-10 \%$ of the trapped protonated proteins. This way, more than $90 \%$ of the photoproduct ions are due to single-photon absorption events. The photoproducts are then extracted into a linear time-of-flight (TOF) mass spectrometer to record a mass spectrum. Mass spectra are recorded in (photons on-photons on-photons off) cycles, to record photoproduct and precursor spectra simultaneously and minimize the influence of fluctuations in ESI signal intensity (for a stable ESI signal, a single "photons off" spectrum at the beginning of an acquisition would be sufficient). The eventual net photoabsorption effect is determined by subtraction of the precursor spectra from the photoproduct spectra, yielding a negative peak at $\mathrm{m} / \mathrm{z}$ of the precursor and positive peaks for the photoproducts. Key features of the experiment are quantitatively reliable mass spectra over the entire relevant $\mathrm{m} / \mathrm{z}$ range as well as absence of He buffer gas during and after the photoexposure. The latter implies that the photoproducts are not cooled.

All mass spectra presented in this article have been obtained with the Groningen tandem mass spectrometer.

\section{The NanoClusterTrap at HZB}

This apparatus is permanently installed at the UE52-PGM beamline. It is a mass spectrometer custom-built to record partial ion yield spectra of trapped cluster and molecular ions $[22,23]$. We have combined the NanoClusterTrap with the Groningen high-fluence ESI source. RF phase space compression, ion guiding, and mass selection are very similar to the Groningen apparatus. The ions then pass an electrostatic quadrupole deflector and are continuously transferred into a linear $\mathrm{RF}$ quadrupole ion trap for storage. The trap content is continuously exposed to the collinear soft X-ray beam, whose intensity is monitored by means of a GaAsP photodiode. The trap is filled with He buffer gas $\left(\sim 1 \times 10^{-4} \mathrm{mbar}\right)$ to facilitate collisional cooling of the photoproducts, with typical temperature equilibration times of less than $1 \mu$ s. The trap can be operated down to $T=3 \mathrm{~K}$ ion trap wall temperature, but is operated at $T \sim 200 \mathrm{~K}$ for the experiments presented here. Bunches of ions are extracted into a reflectron-type TOF mass spectrometer with a repetition rate of the order of $100 \mathrm{~Hz}$. The timing scheme of the experiment only allows for acquisition of a mass range of several hundred daltons at a time. The nominal mass resolution of the instrument is $3000 \mathrm{Da}$, but during the experiments reported here, significantly lower resolutions are employed. The soft X-ray energy is varied with a step size of $100 \mathrm{meV}$ (C and $\mathrm{N} \mathrm{K}$-edge) and $250 \mathrm{meV}$ (O K-edge). The UE52-PGM beamline delivers left or right circularly polarized photons. In general, pairs of mass spectra are recorded for the two polarizations. No polarization effect is observed for melittin within the noise level of the soft X-ray spectra recorded. X-ray absorption spectra are obtained by plotting yields of photoproducts against the photon energy. The data are normalized with respect to the photon flux, measured simultaneously with every absorption spectrum with a GaAsP photodiode at the end of the apparatus. Due to carbon-containing contaminations on the optical elements along the beamline, there is an increased uncertainty of the normalization in the region of the carbon $\mathrm{K}$-edge. The photon energy was calibrated using the nitrogen $\mathrm{K}$-edge of an $\mathrm{N}_{2}$ gas target. The calibration had an error bar of $\pm 125 \mathrm{meV}$.

Key features of the experiments are the large trap capacity, allowing for efficient recording of soft X-ray absorption spectra and the presence of a buffer gas, quenching slow fragmentation channels. No full range and quantitative mass spectra can be obtained.

All soft X-ray spectra presented in this article have been obtained with the NanoClusterTrap.

\section{Electrospray Ionization}

Melittin from honey bee venom was purchased from SigmaAldrich at a $>85 \%$ purity. For the experiments with the Groningen apparatus, a $40-\mu \mathrm{M}$ melittin solution in HPLC-grade methanol is used; $0.005 \%$ formic acid is added to facilitate protonation. For the NanoClusterTrap experiments, a $30-\mu \mathrm{M}$ melittin solution in 44\% HPLC-grade water, 55\% HPLC-grade methanol, and $1 \%$ formic acid is employed. The ESI solution is then pumped through the ESI-emitting needle using a syringe pump with a flow rate of typically less than $10 \mu \mathrm{l} / \mathrm{min}$. After phase space compression in the RF ion funnel, ion currents typically exceed $1 \mathrm{nA}$ and more than $100 \mathrm{pA}$ of mass-selected $[\text { melittin }+3 \mathrm{H}]^{3+}$ ions can be generated.

\section{Results and Discussion}

\section{Soft X-ray Spectra for Non-dissociative Single and Double Ionization}

From a soft X-ray spectroscopy point of view, it is most straightforward to look into the data for non-dissociative single and double photoionization, i.e., the partial ion yields of $[\text { melittin }+\mathrm{qH}]^{(q+1)+}$ and $[\text { melittin }+\mathrm{qH}]^{\left(q^{+} 2\right)+}$ as a function of photon energy. Figure 1 shows the respective spectra for $q=2-4$ at the K-edges of carbon, nitrogen, and oxygen. At all the three edges, the non-dissociative single ionization (NDSI) spectra (Figure 1, top row) exhibit a dominating peak that is due to excitations from the respective $1 \mathrm{~s}$ orbital to the $\pi^{*}$ orbital localized at the $\mathrm{C}=\mathrm{ONH}$ amide group of the protein.

The $\mathrm{C} 1 \mathrm{~s}-\pi_{\mathrm{C}=\mathrm{O}}^{*}$ transition is observed at $288.14 \mathrm{eV}$, at a FWHM of about $0.7 \mathrm{eV}$. Soft X-ray bandwidths of $100 \mathrm{meV}$ $(q=2)$ and $50 \mathrm{meV}(q=3,4)$ were used at the C K-edge. The FWHM is thus mostly due to the variation of the $\mathrm{C} 1 \mathrm{~s}-\pi_{\mathrm{CONH}}^{*}$ transition energies in the different amino acid residues of melittin. The smaller peak at $285.1 \mathrm{eV}$ results from C $1 \mathrm{~s}-\pi^{*}$ transitions in the tryptophan (W) indole sidechain, which is the 


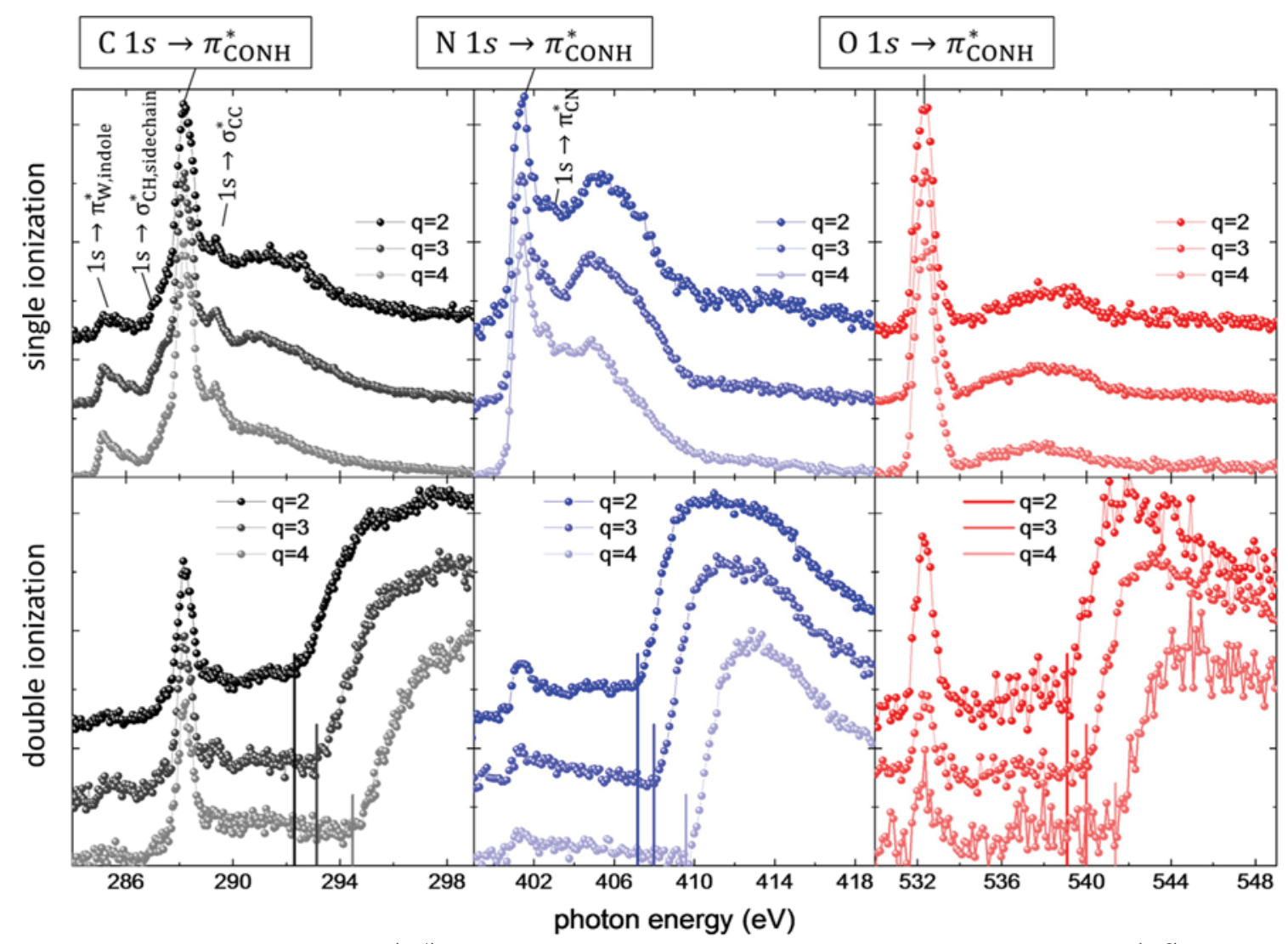

Figure 1. Partial ion yields of $[\text { melittin }+\mathrm{qH}]^{(\mathrm{q}+1)+}$ (non-dissociative single ionization, top row) and [melittin+qH$]^{(\mathrm{q}+2)+}$ (non-dissociative double ionization, bottom row) for $[\text { melittin }+q H]^{q+}$, with $q=2-4$. The three photon energy intervals correspond to the $\mathrm{K}$-edges of carbon (left column), nitrogen (middle column), and oxygen (right column). The single ionization spectra were normalized to the dominating $1 \mathrm{~s}-\pi^{*}$ peak and the double ionization spectra were normalized to the maximum of the photoionization continuum. Offsets of $30 \%$ and $60 \%$ were added to the $q=3$ and $q=4$ spectra for visualization

only aromatic amino acid contained in melittin. The relative intensity of this transition increases strongly with $q$. We have recently shown [21] that this increase is unlikely to be a consequence of the dramatic change in molecular structure upon increasing protonation [20]. It rather reflects a general decrease of protein stability with increasing charge state. The non-dissociative channels discussed here are clearly related to photoabsorption processes that neither deposit sufficient energy for swift thermal fragmentation nor induce fast localized fragmentation. For the most stable protonation case $(q=2)$, NDSI appears not very site specific given the rather broad structureless yield distribution. For the intrinsically less stable cases $(q=3,4)$, absorption in the tryptophan indole group is relatively increased, as $1 \mathrm{~s}-\pi^{*}$ transitions in aromatic sidechains are known to be generally less destructive to protein/peptide integrity [15].

The shoulder at $287.3 \mathrm{eV}$ and the peak at $289.4 \mathrm{eV}$ stem from the $\mathrm{C} 1 \mathrm{~s}-\sigma^{*}(\mathrm{CH})$ and $\mathrm{C} 1 \mathrm{~s}-\sigma^{*}(\mathrm{CC})$ transitions in various sidechains, respectively. All energies are in good agreement with literature data for gas-phase protonated cytochrome $\mathrm{c}$ and neutral amino acids (see Table 1). The broad structure between roughly 290 and $294 \mathrm{eV}$ is due to a superposition of various transitions to $\sigma$-orbitals, shape resonances, and Rydberg states.
With increasing protonation state $q$, this structure gets suppressed, in particular on the high-energy side.

The $\mathrm{N} 1 \mathrm{~s}-\pi_{\mathrm{CONH}}^{*}$ transition is observed at $401.5 \mathrm{eV}$. The $\mathrm{N}$ $1 \mathrm{~s}-\pi_{\mathrm{C}-\mathrm{N}}^{*}$ peak at $402.6 \mathrm{eV}$ is the only additional transition that can be clearly identified. Both energies are close to literature data for protonated cytochrome c (see Table 1). As for C, the broad feature at high energies, starting at $403.5 \mathrm{eV}$ is due to a superposition of various transitions to $\sigma$-orbitals, shape resonances, and Rydberg states. A similar suppression with $q$ as for $\mathrm{C}$ is observed. Comparing the $\mathrm{C}, \mathrm{N}$, and $\mathrm{O} \mathrm{K}$-edge spectra, the broad structure is relatively strongest for $\mathrm{N}$ and its peak intensity reaches about $70 \%$ of the $\mathrm{N} 1 \mathrm{~s}-\pi_{\mathrm{CONH}}^{*}$ peak intensity for $q=2$.

The $\mathrm{O} 1 \mathrm{~s}-\pi_{\mathrm{C}=\mathrm{O}}^{*}$ transition is observed at $532.4 \mathrm{eV}$ which is in good agreement with the values obtained in several studies on gas-phase amino acids and substantially higher than the $531.4 \mathrm{eV}$ reported for cytochrome c (see Table 1). No other peaks are observed. Again, a broad feature is observed which starts at $534 \mathrm{eV}$ and peaks around $538 \mathrm{eV}$. The intensity of this broad feature is weaker than for $\mathrm{N}$ and $\mathrm{C}$ and it only exhibits a slight shift to smaller photon energies with increasing $q$.

The non-dissociative double ionization (NDDI) spectra (Figure 1, bottom row) have an entirely different shape. The 
Table 1. Peak assignments and energies for the $\mathrm{C}, \mathrm{N}$, and $\mathrm{O} \mathrm{K}$-edges from this work, compared to data for gas-phase $[\mathrm{cytochrome} \mathrm{c}+\mathrm{qH}]^{\mathrm{q}^{+}}$and for several neutral gas-phase amino acids. The low-energy peak assigned to tryptophan is a superposition of transitions in the indole sidechain of which only the lowest energy transition can be clearly identified and is reported

\begin{tabular}{|c|c|c|c|c|}
\hline Inner shell & Transition & $\begin{array}{l}{[\text { Melittin }+\mathrm{qH}]^{q+}} \\
(\mathrm{eV})\end{array}$ & $\begin{array}{l}{[\text { Cytochrome c+qH}]^{q^{+}}} \\
(\mathrm{eV})[25]\end{array}$ & Amino acids (eV) \\
\hline \multirow[t]{4}{*}{$\mathrm{C} 1 \mathrm{~s}$} & $1 \mathrm{~s}-\pi^{*}(\mathrm{CONH}$, amide $)$ & $288.14 \pm 0.125$ & 288.3 & $\begin{array}{l}1 \mathrm{~s}-\pi^{*}(\mathrm{CO}) 288.4 \text { (alanine, threonine) [24], } \\
288.4 \text { (tryptophan) [26], } 288.5 \text { (glycine) [27] }\end{array}$ \\
\hline & $\mathrm{C}_{5}-\mathrm{C}_{8} 1 \mathrm{~s}-\pi^{*}{ }_{1}$ (tryptophan) & $285.1 \pm 0.125$ & 285.5 & 285.1 (tryptophan) [26] \\
\hline & $\mathrm{C} 1 \mathrm{~s}-\sigma^{*}(\mathrm{CH})$ & $287.3 \pm 0.125$ & 287.0 & 287.4 (alanine) [24], 287.2 (threonine) [24] \\
\hline & $1 \mathrm{~s}-\sigma^{*}(\mathrm{CC})$ & $289.4 \pm 0.125$ & - & $\begin{array}{l}289.5 \text { (alanine, threonine) [24], } \\
289.4 \text { (glycine) [27] }\end{array}$ \\
\hline \multirow[t]{2}{*}{$\mathrm{N} 1 \mathrm{~s}$} & $1 \mathrm{~s}-\pi^{*}(\mathrm{CONH}$, amide $)$ & $401.5 \pm 0.125$ & 401.4 & 401.2 (alanine [24], glycine), 401.3 (threonine) [24] \\
\hline & $1 \mathrm{~s}-\pi^{*}(\mathrm{CN})$ & $402.6 \pm 0.125$ & 403.2 & 402.4 (alanine [24], glycine), 402.5 (threonine) [24] \\
\hline $\mathrm{O} 1 \mathrm{~s}$ & $1 \mathrm{~s}-\pi^{*}(\mathrm{CONH}$, amide $)$ & $532.4 \pm 0.125$ & 531.4 & $\begin{array}{l}1 \mathrm{~s}-\pi^{*}(\mathrm{CO}) 532.2 \text { (glycine) [27], } \\
532.2 \text { (alanine) [24], 532.1 (threonine) [24], } 532.1 \text { (tryptophan) [26] }\end{array}$ \\
\hline
\end{tabular}

main feature of the NDDI spectra, the broad structure at the highest energies is due to direct $1 \mathrm{~s}$ ionization followed by Auger decay. For carbon, nitrogen, and oxygen, this manifests as an almost linear increase from the 1s excitation background, followed by a broad ionization continuum. The $1 \mathrm{~s}$ ionization energy can be determined from the onset of the linear section [16]. All 1s ionization energies exhibit a pronounced increase with protonation state (see Table 2). 1s ionization energies of many neutral amino acids have been determined by X-ray photoemission spectroscopy (XPS) in the Prince group and the lowest values are systematically lower than the ionization energies presented here [24]. For the typical example of alanine, the values are added in Table 1.

For ubiquitin in protonation states ranging from 4 to 11 , Milosavljević et al. have recently linked the increase of $\mathrm{C} 1 \mathrm{~s}$ ionization energy to the protein tertiary structure [16]. For $q=5-$ 8 , no clear increase in $1 \mathrm{~s}$ ionization energy $(294.3 \mathrm{eV})$ was observed which was explained by unfolding of the protein in this range of protonation states that strongly influences the effective Coulomb field of the molecule. In contrast, the 3D structure of gas-phase protonated melittin is mostly a secondary structure. For this system, hydrogen/deuterium exchange and ion mobility studies provide evidence for strong structural relaxation in the range of $q=2-4$, with melittin being predominantly helical at $q=2$ and relaxed at $q=4[20,28]$. Our data shows a systematic increase in $\mathrm{C}, \mathrm{N}$, and $\mathrm{O} 1 \mathrm{~s}$ ionization energies for $q=2-4$, which accompanies the loss in secondary structure.

For photon energies below the ionization threshold, for all the three K-edges, the dominant NDSI peak $\left(1 \mathrm{~s}-\pi_{\mathrm{C}=\mathrm{O}}^{*}\right)$ is

Table 2. $[\text { Melittin }+\mathrm{qH}]^{q^{+}}$detachment energies for the 1s levels of carbon, nitrogen, and oxygen

\begin{tabular}{llll}
\hline $\begin{array}{l}\text { Protonation } \\
\text { state } q\end{array}$ & $\begin{array}{l}\text { C 1s detachment } \\
\text { energy (eV) }\end{array}$ & $\begin{array}{l}\text { N 1s detachment } \\
\text { energy (eV) }\end{array}$ & $\begin{array}{l}\text { O 1s detachment } \\
\text { energy (eV) }\end{array}$ \\
\hline 2 & 292.2 & 407.2 & 539.1 \\
3 & 293.1 & 408.0 & 540.4 \\
4 & 294.5 & 409.6 & 541.4 \\
Neutral & 291 & 405.2 & 538.2 \\
\hline
\end{tabular}

observed but at reduced relative intensity, as are the other features of the NDSI spectra. This is in line with the notion that the most probable process following $1 \mathrm{~s}$ excitation is a resonant Auger decay, in which a valence electron fills the 1s vacancy. The simultaneous emission of an Auger electron from the same site leads to a singly ionized molecule. Two-electron (or more) Auger processes typically occur with much larger probability [29]. These processes lead to double ionization upon resonant $1 \mathrm{~s}$ excitation, as visible in the bottom panels of Figure 1. Two-electron Auger processes do not, however, explain the dependence of the latter channel on protein protonation state and therefore on 3D structure.

In our recent study on melittin $\mathrm{C} \mathrm{K}$-edge photoionization at cryogenic temperatures [21], we have already shown that double ionization following $1 \mathrm{~s}$ excitation can also be due to electron impact ionization "from within." In this process, the Auger electron emitted during the de-excitation of the initial photoinduced $1 \mathrm{~s}$ vacancy induces an electron impact ionization event elsewhere in the molecule. The cross section for this process can be estimated from atomic cross sections for electron impact ionization. To this end, we have used a Monte Carlo approach in which for each $\mathrm{K}$-edge $(\mathrm{C}, \mathrm{N}$, and $\mathrm{O})$, a typical Auger electron energy is chosen $(250 \mathrm{eV}, 375 \mathrm{eV}$, and $500 \mathrm{eV}$, respectively). Clearly, this is a strong simplification, as the actual Auger electron spectra are likely very different for the different $\mathrm{K}$-edges. For each K-edge, we have then used the tabulated electron atomic impact cross sections for the $\mathrm{H}, \mathrm{C}, \mathrm{N}$, and $\mathrm{O}$ constituents of melittin $[30,31]$. For a given 1 s photoexcited atomic site in the $\mathrm{CONH}$ groups along the protein backbone, we have then computed $2 \times 10^{5}$ random straight line trajectories. For each trajectory we computed whether or not it leads to an ionization event before the Auger electron leaves the protein. This approach is loosely based on the independent atom model (IAM), often employed in electron scattering from molecular systems $[32,33]$. Garcia and co-workers have shown that for an electron energy of $300 \mathrm{eV}$, corrections to IAM scattering cross sections for $\mathrm{CO}_{2}$ are smaller than $10 \%[32,33]$. For higher energies, atomic electron impact ionization cross sections decrease and the accuracy of the IAM should be even better. As a last step, we then average the ionization probability for all 
carbon, nitrogen, and oxygen amide sites, respectively (under the approximation that the $1 \mathrm{~s}-\pi^{*}(\mathrm{CONH} / \mathrm{amide})$ photoexcitation cross section is the same everywhere along the protein backbone.

To show the influence of protein conformation on this channel, we have performed the Monte Carlo calculations for the melittin structure in solution that features a very high $\alpha$ helical content and for a hypothetical linear configuration. Table 3 shows the results. For all the three edges, the simulations yield a substantially lower contribution of ionization "from within" for the linear conformation $(33 \%, 26 \%$, and $20 \%$ for carbon, nitrogen, and oxygen, respectively) as compared to the helical conformation $(44 \%, 39 \%$, and $27 \%$ for carbon, nitrogen, and oxygen, respectively). Table 3 also shows the relative contribution of $1 \mathrm{~s}-\pi_{\mathrm{CONH}}^{*}$ excitation with respect to the maximum of the ionization continuum for NDDI from Figure 3. The experimentally observed ratio for maximum helicity $(q=2)$ and minimum helicity $(q=4)$ is barely larger than 1 for carbon (1.04), while for nitrogen and oxygen, the ratios are much larger than 1, 1.67, and 2.29 respectively. For a medium-sized protein such as melittin, the quantitative difference between simulation and experimental data is most likely due to the fact that the nondissociative ionization channels are competing with various fragmentation channels. The respective branching ratios depend for instance on protonation state, photoabsorption site (carbon, nitrogen, or oxygen), and internal temperature. In our previous study on soft X-ray spectroscopy on melittin at cryogenic temperatures $(T=10 \mathrm{~K})$, much stronger nondissociative ionization from within was observed for the melittin C K-edge [21]. At a temperature of $10 \mathrm{~K}$, the photoabsorption occurs in a molecule with almost zero internal energy, whereas at room temperature, the thermal energy amounts to about $5 \mathrm{eV}$ [18]. This implies a significantly smaller relative yield of non-dissociative processes for the experiments at room temperature, in particular for the ionization-from-within events, whose contribution to nondissociative double ionization will be quenched. In the same context, Milosavljević and co-workers investigated the influence of the protonation state on $\mathrm{C} \mathrm{K}$-edge soft X-ray absorption for the much larger protonated protein ubiquitin and observed a substantial reduction of the $\mathrm{C} 1 \mathrm{~s}-\pi_{\mathrm{CONH}}^{*}$ resonance in NDSI with decreasing protonation state but did not assign a mechanism [16].

\section{Small Neutral Losses}

Depending on the final electronic state of the inner-shell excitation process and even more so, on the molecular orbitals involved in the subsequent Auger de-excitation, the electronic excitation of the melittin cations can cover a range of $50 \mathrm{eV}$ or more, with an average of almost $20 \mathrm{eV}[18,34]$.

Even when neglecting possible direct fragmentation channels, for instance due to population of repulsive states, innershell excitation processes will thus ultimately lead to fragmentation of the cationic protein, for example losses of small neutral groups. In the mass spectra, losses of a small neutral group manifest as peaks on the low mass side of the NDSI and NDDI peaks. This is illustrated for NDSI of $[\text { melittin }+3 \mathrm{H}]^{3+}$ in the mass spectrum in Figure 2(a)). On the low mass side of the $[\text { melittin }+3 \mathrm{H}]^{4+}$ peak, additional peaks corresponding to the loss of neutrals with $m=17,29,44$, and $59 \mathrm{Da}$ are observed:

- $m=17$ Da: loss of an ammonia $\left(\mathrm{NH}_{3}\right)$ group, either from residues whose sidechain contains an $\mathrm{N}$ atom (here $\mathrm{Q}, \mathrm{K}$, $\mathrm{R}$, and $\mathrm{W}$ ) or from the amidated C-terminal. For CID, it was shown that both types of losses can compete [35]. For VUV photofragmentation of protonated substance $\mathrm{P}$, Canon et al. have assigned $\mathrm{NH}_{3}$ loss to originate primarily from the $\mathrm{K}$ residue [36].

- $m=29 \mathrm{Da}$ : loss of $\mathrm{C}_{2} \mathrm{H}_{5}$ from I residues has been observed in CID [37]. Another possibility would be $\mathrm{CH}_{3} \mathrm{~N}$ loss from $\mathrm{R}$.

$-m=44$ Da: loss of $\mathrm{CH}_{4} \mathrm{~N}_{2}$ from the $\mathrm{R}$ residue has been observed in ECD [38] and VUV photoionization [36].

- $m=59 \mathrm{Da}$ : loss of $\mathrm{C}_{2} \mathrm{H}_{5} \mathrm{NO}$ from $\mathrm{Q}$ and $\mathrm{CH}_{5} \mathrm{~N}_{3}$ from $\mathrm{R}$ did show up in ECD [38]. For VUV photons, the latter channel was observed [36].

While all four neutral loss peaks are quite intense in the absence of a buffer gas in the ion trap, the presence of helium buffer gas substantially quenches all but the $m=44\left(\mathrm{CH}_{4} \mathrm{~N}_{2}\right)$ loss peak. This implies that $\mathrm{CH}_{4} \mathrm{~N}_{2}$ loss from $\mathrm{R}$ is associated either to the lowest activation energy or to the fastest dissociation rate, allowing for the process to occur before the protein excess energy thermalizes with the buffer gas. For the case of double ionization without the presence of buffer gas, both NDDI and double ionization with small neutral loss are fully quenched. In the presence of He buffer gas, no neutral loss channels besides $m=44$ loss can be clearly distinguished. The

Table 3. Monte Carlo results for the probabilities for a typical Auger electron emitted from amide carbon, nitrogen, and oxygen, to ionize an atomic site within the protein. Linear refers to an idealized linear configuration, and helical refers to the solution geometry obtained from the protein database. The right columns give the relative intensities of the $1 \mathrm{~s}-\pi_{\mathrm{CONH}}^{*}$ resonance with respect to the maximum of the $1 \mathrm{~s}$ ionization continuum for $q=2-4$ and the ratio between the values for $q=2$ (helical) and $q=4$ (non-helical)

\begin{tabular}{lllllll}
\hline & Linear (\%) & Helical (\%) & Ratio of hel to lin & $q=2$ & $q=3$ & $q=4$ \\
\hline C 1s Auger $(250 \mathrm{eV})$ & 33 & 44 & 1.35 & 0.7 & 0.68 \\
N 1s Auger $(375 \mathrm{eV})$ & 26 & 39 & 1.5 & 0.25 & 0.2 \\
O 1s Auger $(500 \mathrm{eV})$ & 20 & 27 & 1.34 & 0.8 & 0.42 & 0.15 \\
\hline
\end{tabular}


x3

(a)
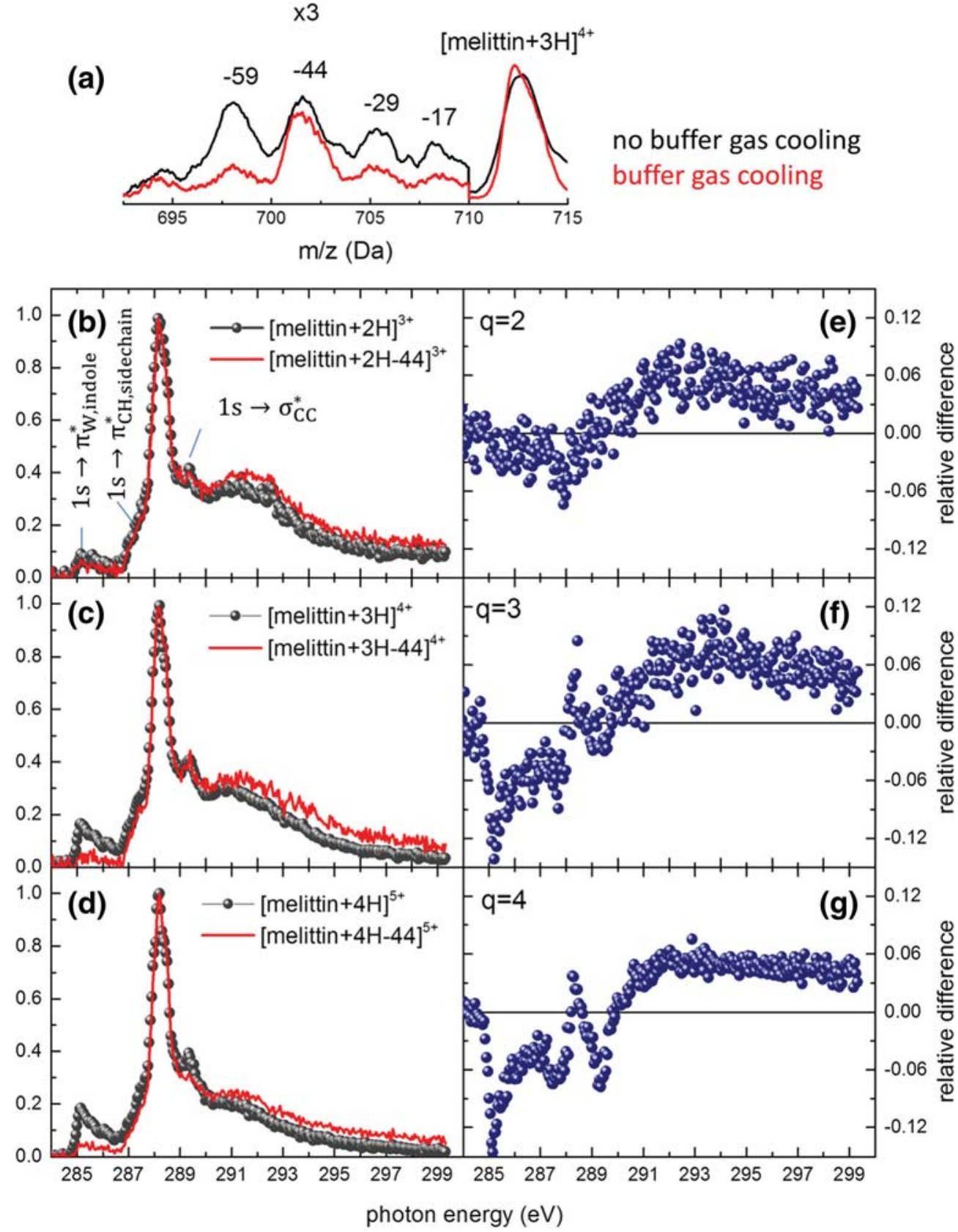

Figure 2. (a) Zoom into the soft X-ray photoionization mass spectrum at $288.14 \mathrm{eV}$. The NDSI peak is observed at $712.6 \mathrm{Da}$. The $\mathrm{m} / \mathrm{z}$ region of the neutral loss peaks $(m=692.5-720 \mathrm{Da})$ is multiplied by 3. (b-d) Normalized partial ion yields of [melittin+qH] $]^{(\mathbf{q}+1)+}(\mathrm{NDSI}$, gray symbols) and [melittin+qH-44$]^{(\mathrm{q}+1)+}$ (single ionization with loss of $44 \mathrm{Da}$, red line) for [melittin+qH$]^{\mathrm{q}+}$, with $q=2-4$. (e-g) Relative differences between the normalized spectra

following paragraphs on neutral losses will thus focus on the $m=44$ loss channel.

Figure 2(b-d) shows the partial ions yields for the $m=44 \mathrm{Da}$ loss channel [melittin $+\mathrm{qH}-44]^{(q+1)+}$ with the NDSI yield $[\text { melittin }+\mathrm{qH}]^{(q+1)+}$ at the $\mathrm{C} \mathrm{K}$-edge for $q=2-4$. All spectra are normalized to the peak at $288.14 \mathrm{eV}$. Figure 2(e-g) displays the respective difference spectra of both channels. For $q=2-4$, the broad spectral feature starting at $290 \mathrm{eV}$ is systematically higher for the $m=44$ loss channel. This is intuitively clear, as $1 \mathrm{~s}$ excitation to higher excited states implies higher excitation energies. The $1 \mathrm{~s}-\pi_{\mathrm{w}, \text { indole }}$ transition is the lowest energy feature at the $\mathrm{C} \mathrm{K}$-edge. As discussed in the previous section, this transition is particularly strong in the NDSI spectra for $q=3$ and 4 . This extraordinary strength of the transition is lifted for the $m=44$ loss and similar relative intensities of the $1 \mathrm{~s}-\pi_{\mathrm{w} \text {,indole }}$ transition are observed for all three protonation states. Furthermore, the $1 \mathrm{~s}-\sigma_{\mathrm{CC}}$ transition is reduced for the $m=44$ loss channel and $q=4$.

The soft X-ray partial ion yield spectra for $m=44\left(\mathrm{CH}_{4} \mathrm{~N}_{2}\right)$ loss from doubly ionized $[\text { melittin }+\mathrm{qH}]^{(\mathrm{q}+2)+}$ are shown in 


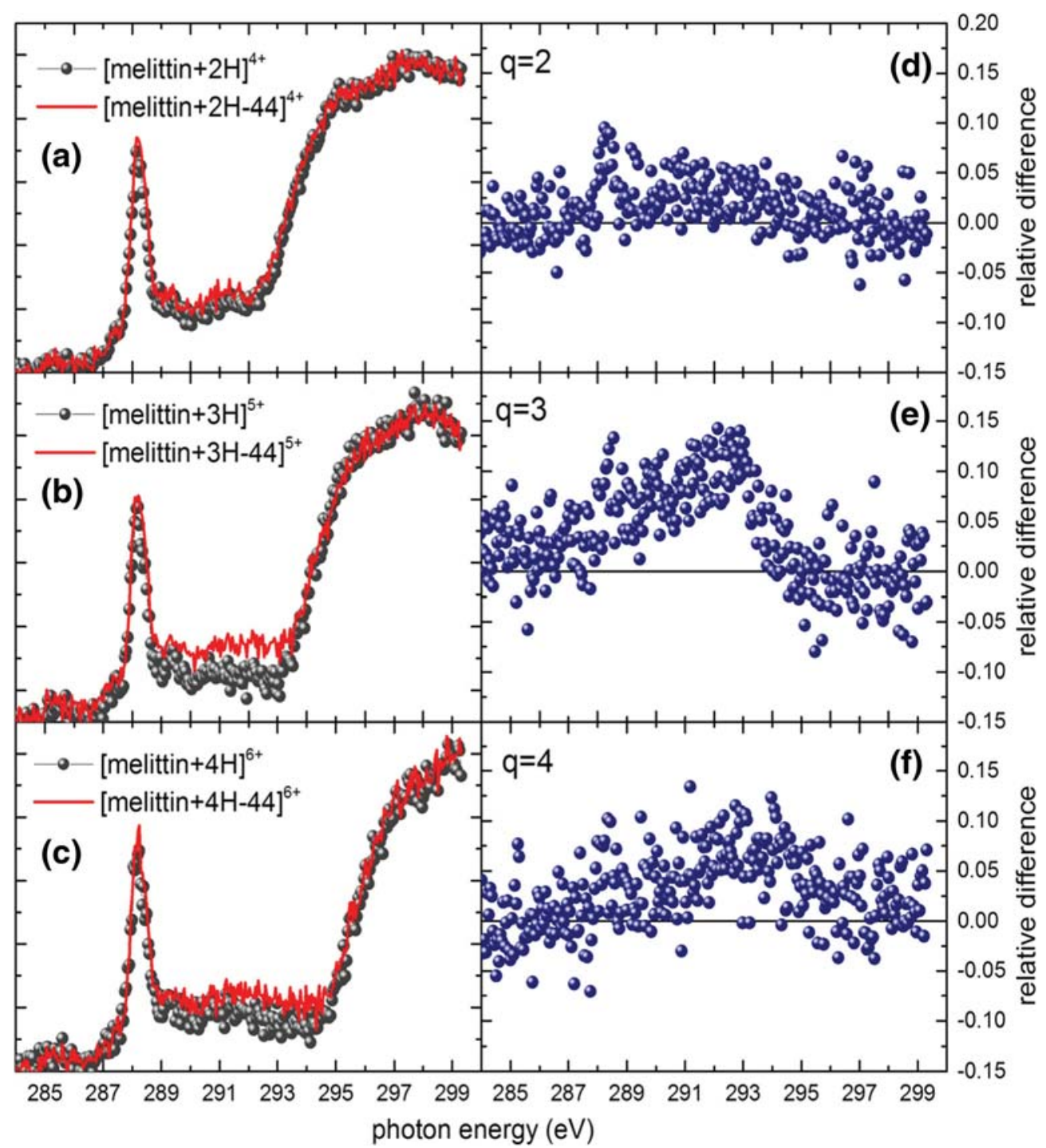

Figure 3. (a-c) Normalized partial ion yields of [melittin $+\mathrm{qH}]^{(\mathrm{q}+2)+}\left(\mathrm{NDDI}\right.$, gray symbols) and $[\text { melittin+qH}-44]^{(\mathrm{q}+2)+}(\mathrm{double}$ ionization with loss of $44 \mathrm{Da}$, red line) for $[\text { melittin }+\mathrm{qH}]^{\mathrm{q}}$, with $q=2-4$. (d-f) Relative differences between the normalized spectra

Figure 3. The left column compares the NDDI channel (gray symbols) with the respective $m=44$ loss (red solid line, all spectra are normalized to the double ionization maximum), while the right column displays the difference between both spectra. Clearly, for all protonation states $q=2-4$, the differences are moderate and limited to energies below the ionization threshold. For double protonation, the melittin is most stable and almost identical soft X-ray spectra are observed whereas for higher protonation states $(q=3,4)$, the spectrum for the $m=44$ loss channel exhibits higher intensities between the main $1 \mathrm{~s}-\pi^{*}$ resonance and the onset of double ionization. As mentioned before, this energy region represents $1 \mathrm{~s}$ excitation into higher excited $\sigma$-states and Rydberg states, whose population seem to favor neutral losses.

\section{Formation of Sequence Ions}

The mass spectrometric most interesting features of the spectra are the peaks due to protein backbone scission. These so-called sequence ions are dictated by the primary structure of the molecule. Canon et al. have already used synchrotron radiation to study the dependence of sequence ion formation on the vacuum ultraviolet photon energy, for a number of proteins [41].

The data for doubly protonated $[\text { melittin }+2 \mathrm{H}]^{2+}$ at the $\mathrm{C} \mathrm{K}$ edge are displayed in Figure 4. The panel on the bottom shows two mass spectra obtained with the Groningen tandem mass spectrometer without helium buffer gas. The photon energies $288.3 \mathrm{eV}$ and $296 \mathrm{eV}$ correspond to the $\mathrm{C} 1 \mathrm{~s}-\pi^{*}$ excitation in the amide $\mathrm{C}=\mathrm{O}$ group and to direct $1 \mathrm{~s}$ ionization, respectively. A large number of fragment peaks is apparent but only a few of these peaks have high intensity when measured with the NanoClusterTrap in the presence of buffer gas. For instance, the peaks at 812 and 826 are not observed with buffer gas and most likely product ions from slow fragmentation processes.

The four panels in Figure 4 show the normalized partial ion yields for four unambigously identified singly charged 


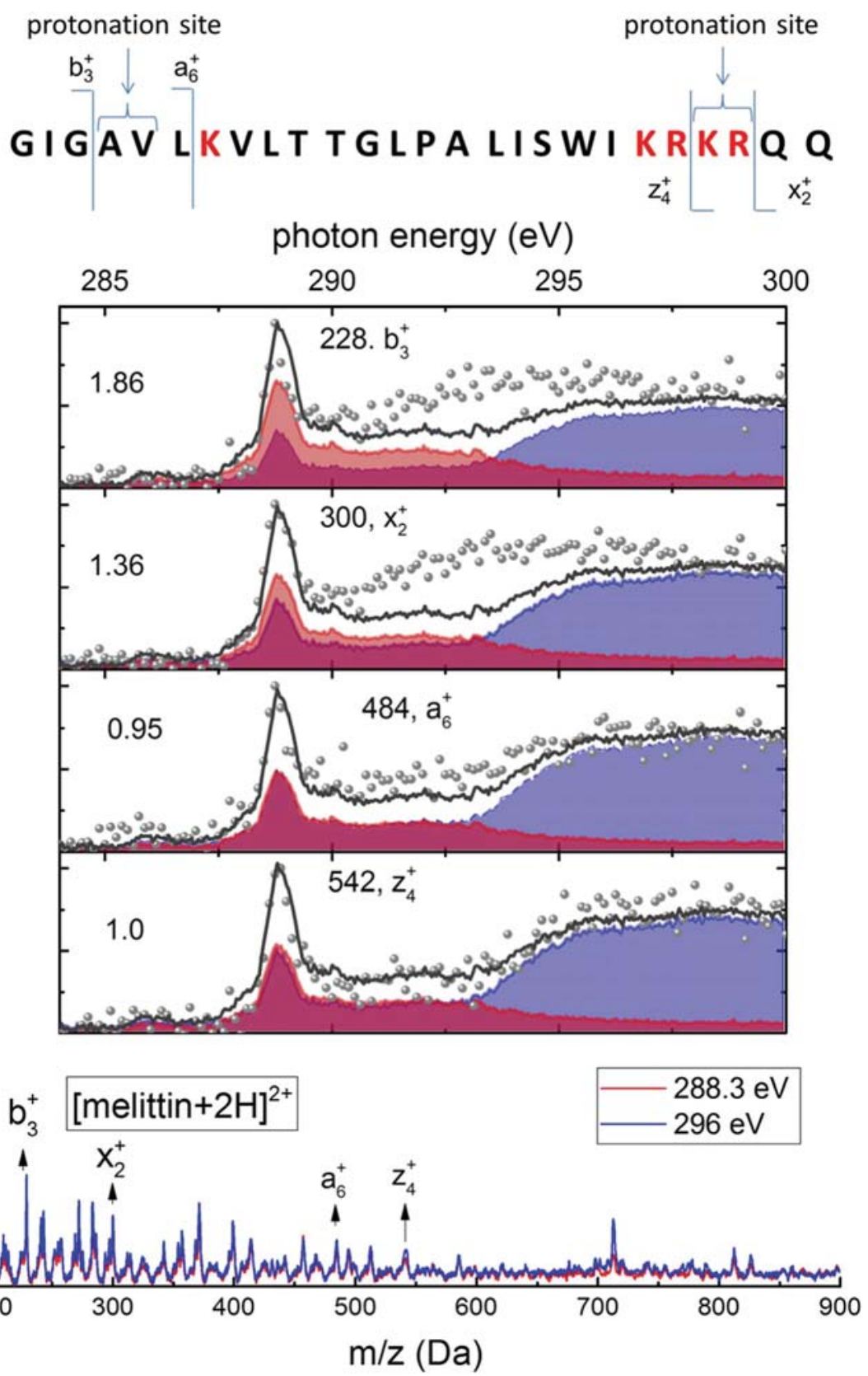

Figure 4. Bottom: mass spectra for [melittin+2H] ${ }^{2+}$ photoionization at $288.3 \mathrm{eV}\left(\mathrm{C}\right.$ 1s- $\pi^{*}$ excitation) and $296 \mathrm{eV}(\mathrm{C}$ inner-shell ionization) recorded without buffer gas. Soft X-ray spectra: partial ion yields (gray circles) as a function of C K-edge photon energy for the four sequence ions, labeled in the mass spectra. The red filled spectrum is the fitted NDSI data and the blue filled spectrum is the fitted NDDI data. The black solid line is the sum of NDSI and NDDI. On each panel, the ratio between the peak maxima of NDSI and NDDI contributions is given. Top: melittin sequence with highest proton affinity residues marked red. The protonation sites were identified in $[39,40]$

fragments as a function of photon energy at the C K-edge (for additional fragments, see the supplementary information). Each spectrum was fitted by the sum of the NDSI (red) and NDDI (blue) spectra from Figure 1. The total fit is given as a solid black line. On a first glance, it is obvious that all four fragments (as well as the other fragments in the mass spectrum) have strong contributions from both, single and double ionization, i.e., no strong final charge-state selectivity is observed. This is in line with the expectations, as charge is not a key issue for the stability of the doubly protonated precursor. Deviations between fit and data points are generally limited to the 290 $295-\mathrm{eV}$ region, where experimental data is often markedly higher than the fit. The following discussion is based on the assumption that these deviations are due to larger contributions of $1 \mathrm{~s}$ excitation into energetically higher unoccupied orbitals, i.e., higher electronic excitation. The electronic excitation upon direct $1 \mathrm{~s}$ photoionization is a priori independent of photon energy. 


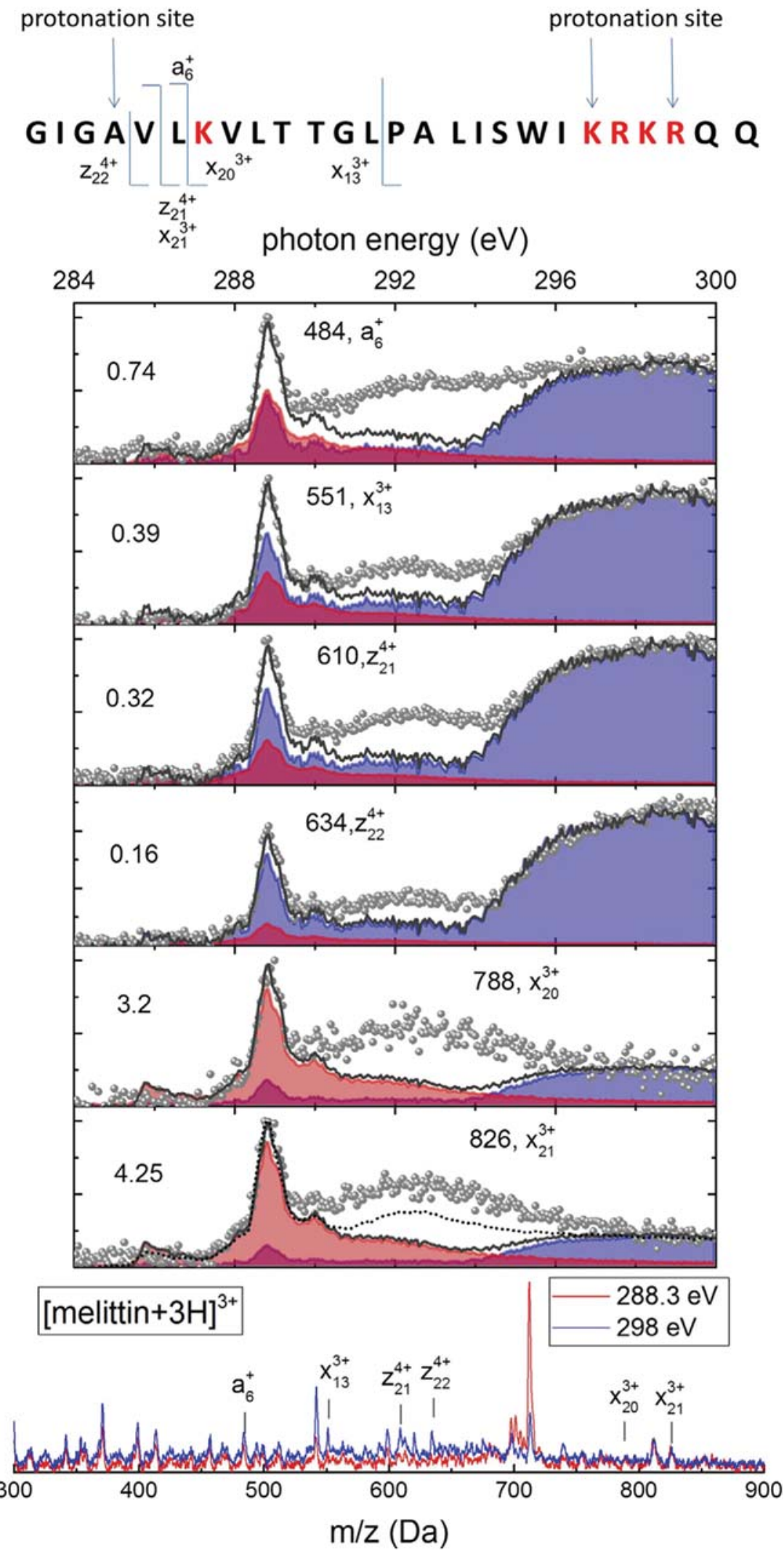

Figure 5. Bottom: mass spectra for $[\text { melittin }+3 \mathrm{H}]^{3+}$ photoionization at $288.3 \mathrm{eV}\left(\mathrm{C} 1 \mathrm{~s}-\pi^{\star}\right.$ excitation) and $298 \mathrm{eV}$ (C inner-shell ionization) recorded without buffer gas. Soft X-ray spectra: partial ions yields as a function of $\mathrm{C}$ K-edge photon energy for the six sequence ions, as labeled in the mass spectra (gray circles). The red filled spectrum always is the fitted NDSI data and the blue filled spectrum is the fitted NDDI data. The black solid line is the sum of NDSI and NDDI. On each panel, the ratio between the peak maxima of NDSI and NDDI contributions is given. Top: melittin sequence with highest proton affinity residues in red. The protonation sites were identified in [39, 40] 
For two fragments $\left(\mathrm{a}_{6}^{+} ; \mathrm{z}_{4}^{+}\right)$, the fit is excellent and deviations are negligible. Partial ion yields that are well reproduced by a superposition of non-dissociative single and double ionization yields most likely correspond to activation energies as low as those of the non-dissociative channels. The activation energies are likely lower than for $m=44$ loss following single ionization, where higher excited states are favored (Figure 2(b, e)). This can be explained with the help of the melittin sequence depicted on the top of Figure 4, where the five $\mathrm{K}$ and $\mathrm{R}$ residues, the residues with the highest proton affinity, are marked in red. The first two protonation sites in melittin have been determined by electron capture dissociation [39] to lie between $A_{4}$ and $V_{5}$ and between $\mathrm{K}_{23}$ and $\mathrm{R}_{24}$, with the first site likely stabilized by the helical secondary structure of the protein. As expected, the low activation energy fragments $\mathrm{a}_{6}{ }^{+}$and $\mathrm{z}_{4}{ }^{+}$both involve scissions that are close to the protonation sites. A deeper look into the data (see Figure $\mathrm{S} 1$ ) indicates that the protonation site is actually $\mathrm{K}_{7}$ rather than $\mathrm{A}_{4} / \mathrm{V}_{5}$.

Is there a similar relationship between activation energy for protein backbone scission and characteristics of the respective fragment soft X-ray spectrum also present at higher protonation states? Figure 5 shows the respective data for triply protonated $[\text { melittin }+3 \mathrm{H}]^{3+}$. Again, only a selection of unambiguously assigned fragments is shown (for a more extensive list, see the supplementary information). A first glance at the spectra reveals that there are still sequence ions such as $\mathrm{a}_{6}^{+}$, which display strong contributions, here with an NDSI/NDDI ratio of $0.74 . \mathrm{a}_{6}{ }^{+}$is observed for $[\text {melittin }+2 \mathrm{H}]^{2+}$ as well. However, the NDSI/NDDI sum fit is not as perfect as observed for the $[\text { melittin }+2 \mathrm{H}]^{2+}$ case (see Figure 4). Deviations are again starting at $289 \mathrm{eV}$ and now extending even beyond $295 \mathrm{eV}$, probably because of the higher ionization energy.

Other sequence ions result primarily from single ionization $\left(\mathrm{x}_{20}{ }^{3+}\right.$ and $\left.\mathrm{x}_{21}{ }^{3+}\right)$ with NDSI/NDDI ratios (determined from the peak maxima) of 3.2 and $4.25 . \mathrm{x}_{21}{ }^{3+}$ is observed in the mass spectra for doubly protonated melittin in Figure 4, which are obtained without a buffer gas but not in the presence of a buffer gas. For $[\text { melittin }+3 \mathrm{H}]^{3+}$, this channel has a much faster dissociation rates than for $[\text { melittin }+2 \mathrm{H}]^{2+}$, possibly due to the additional Coulomb repulsion between $\mathrm{x}_{21}{ }^{3+}$ and the complementary fragment which is now singly charged. Surprisingly, for these channels, the deviations between fit and actual data are largest, indicating high activation energies.

A third class of sequence ions $\left(\mathrm{x}_{13}{ }^{3+}, \mathrm{z}_{21}{ }^{4+}\right.$, and $\left.\mathrm{z}_{22}{ }^{4+}\right)$ are dominated by double ionization with NDSI/NDDI ratios between 0.16 and 0.39 . Here, deviations are smallest and accordingly, activation energies are lowest. For $[\text { melittin }+3 \mathrm{H}]^{3+}$, protonation sites were determined by Morrison et al. using UV photodissociation as $\mathrm{A}_{4}, \mathrm{~K}_{21}$, and $\mathrm{R}_{24}$ [40]. The fragments $\mathrm{Z}_{21}{ }^{4+}$ and $\mathrm{z}_{22}{ }^{4+}$ are close to the $\mathrm{A}_{4}$ site. As these two fragments are predominantly formed after double ionization, the remaining $\mathrm{N}$-terminal fragment carries a protonation site and has to be singly charged. Fragment $\mathrm{x}_{13}{ }^{3+}$ is not close to one of the protonation sites. Instead, the scission is on the $\mathrm{N}$-side of the only proline $\left(\mathrm{P}_{14}\right)$ residue. Kjeldsen et al. have identified the fourth protonation site in this region [39]. Also, an enhancement of scission probability close to $\mathrm{P}$ residues is often observed [42].

Figure 6 displays the dataset for $[\text { melittin }+4 \mathrm{H}]^{4+}$. The protonation states are difficult to assign on the basis of existing literature [39, 40]. Most likely, two protons are located in the high proton affinity KRKR region, a third proton sits in the vicinity of the $A_{4}$ residue and the fourth is located in the general region of the $\mathrm{P}_{14}$ residue. Again, three groups of peaks can be identified. Fragments $\mathrm{a}_{6}{ }^{+}$and $\mathrm{x}_{13}{ }^{4+}$ feature comparable contributions from single and double ionization. Their NDSI/NDDI ratio is between 0.71 and 1.1 , respectively. Deviations between the fit and the experimental data are generally even larger than for $[\text { melittin }+3 \mathrm{H}]^{3+}$.

The fragment $\mathrm{a}_{6}{ }^{+}$has been discussed for the two lower protonation states and it is likely related to a multiple scission scenario.

$\mathrm{Z}_{22}{ }^{4+}$ is dominated by single ionization with a ratio of 5.9. Note, that for the triply protonated precursor, $\mathrm{z}_{22}{ }^{4+}$ was almost solely formed by double ionization (see Figure 5). Also, this fragments exhibits large differences between fit and experimental data and probably involves high activation energies.

The last set of fragments $\left(\mathrm{b}_{7}{ }^{2+}, \mathrm{y}_{13}{ }^{4+}\right.$, and $\left.\mathrm{z}_{22}{ }^{5+}\right)$ is dominated either by double ionization or for $b_{7}{ }^{2+}$ even solely due to double ionization. For these fragments, the agreement between fit and experimental data is remarkably good and it is almost perfect for fragment $b_{7}{ }^{2+}$. This hints at very low activation energies for the fragments. Double ionization of $[\text { melittin }+4 \mathrm{H}]^{4+}$ yields a sixfold charged ion, which probably is the maximum charge this protein can stabilize [43]. Accordingly, activation energies for this system are most likely very low.

\section{Conclusions}

We have performed a soft X-ray absorption spectroscopy case study of melittin. Three main classes of reaction channels were investigated: non-dissociative ionization, loss of small neutral molecules, and formation of backbone scission ions.

The analysis of the non-dissociative ionization yields shows clear evidence for significant double ionization in melittin below the ionization threshold. This double ionization is to a large extent due to electron impact ionization by Auger electrons emitted after soft X-ray absorption. We observe a clear decline of the respective yield with decreasing melittin protonation state. There are two major contributions to this trend. First of all, the less compact structure for higher charge states entails lower yields of secondary ionization events triggered by the emitted Auger electrons. This is in line with Monte Carlo simulations of secondary electron ionization in the framework of the independent atom model (IAM).

The second class of reaction channels under investigation is small neutral molecule losses. We observe $-17,-29,-44$, and -59 loss peaks which can be assigned to loss of $\mathrm{NH}_{3}, \mathrm{C}_{2} \mathrm{H}_{5}$ $\mathrm{CH}_{4} \mathrm{~N}_{2}$, and $\mathrm{C}_{2} \mathrm{H}_{5} \mathrm{NO}$ groups from amino acid residues in the peptide. -17 can be assigned to lysine $(\mathrm{K}),-29$ to isoleucine (I), and -44 and -59 to arginine (R). Interestingly, the presence 

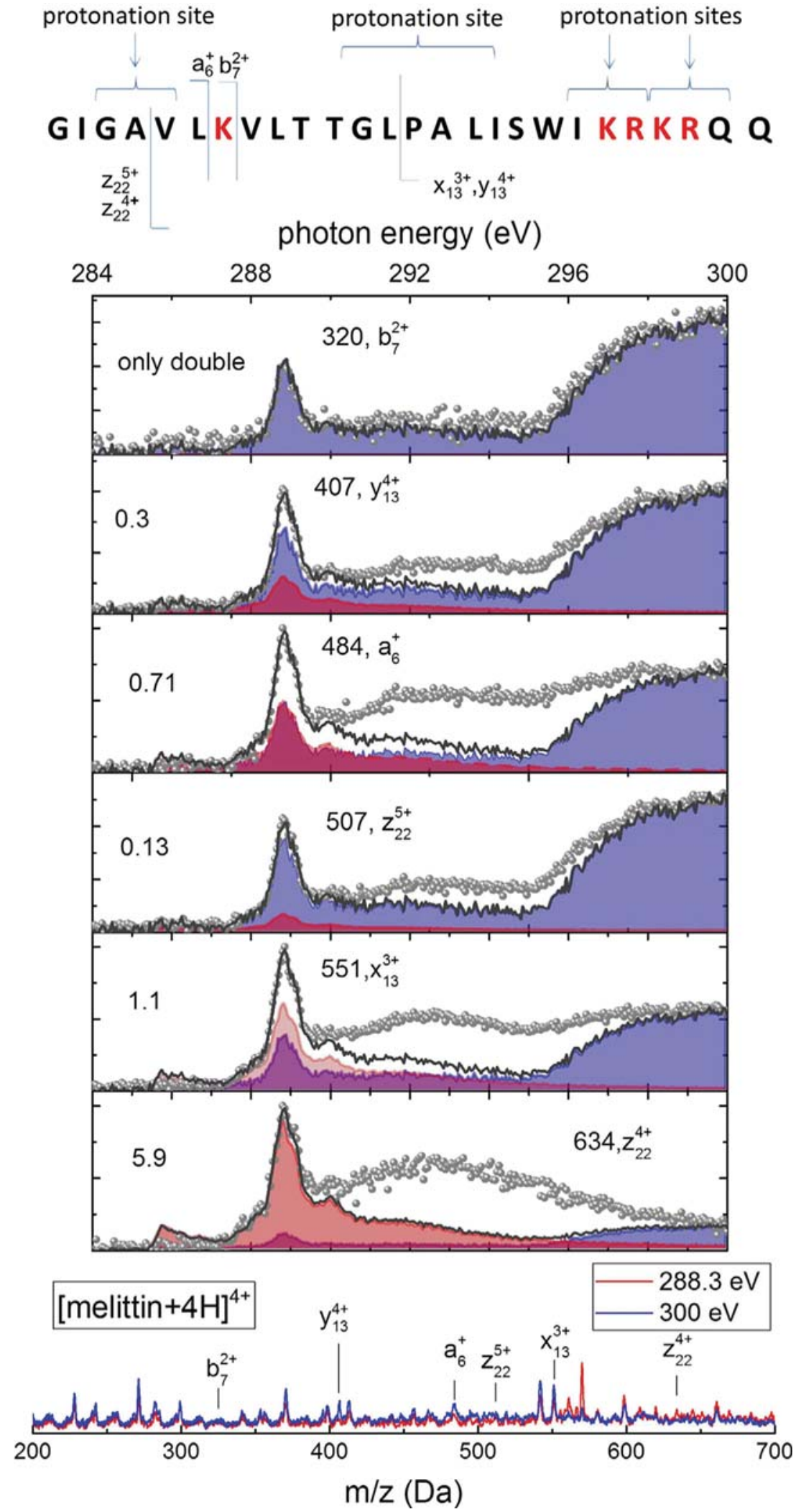

Figure 6. Bottom: mass spectra for [melittin+4H] $]^{4+}$ photoionization at $288.3 \mathrm{eV}\left(\mathrm{C}\right.$ 1s- $\pi^{\star}$ excitation) and $300 \mathrm{eV}(\mathrm{C}$ inner-shell ionization) recorded without buffer gas. Soft X-ray spectra: partial ions yields as a function of C K-edge photon energy for the six sequence ions, as labeled in the mass spectra (gray circles). The red filled spectrum always is the fitted NDSI data and the blue filled spectrum is the fitted NDDI data. The black solid line is the sum of NDSI and NDDI. On each panel, the ratio between the peak maxima of NDSI and NDDI contributions is given. Top: melittin sequence with highest proton affinity residues in red. The protonation sites were identified in [39, 40] 
of buffer gas during photoexposure leads to quenching of all these channels, with the exception of -44 loss. Most likely, this channel has the lowest activation energy or a fast dissociation rate.

For sequence ions stemming from backbone scission, the respective partial ion yields were fitted with a superposition of non-dissociative single (NDSI) and double ionization (NDDI) spectra. The ratio between NDSI and NDDI in the fit is defining the relative importance of single and double ionization for fragment formation. The deviations between the NDSI+NDDI fit and fragment yield dependencies from photon energy are mainly observed in the 290-295-eV energy range, where experimental data was exceeding the fit for some fragments. This deviation can be explained by the higher activation energy required for formation of these fragments.

With sufficient mass resolution, the combination of soft Xray spectroscopy and mass spectrometry allows to assign a specific X-ray absorption spectrum to each ion in the mass spectrum. This spectrum can be used to study the energy deposition for the respective fragmentation and determine the number of removed electrons.

\section{Funding Information}

We thank HZB for the allocation of synchrotron radiation beamtime. The research leading to these results has received funding from the European Community's Seventh Framework Programme (FP7/2007-2013) under grant agreement no. 312284 and from the BMBF under project number 05K16VF1. S. B., S. Dö., and S. De. were supported by the Helmholtz Initiative and Networking Fund through the Young Investigator Group Program. S.B. and S.T. acknowledge support from the Deutsche Forschungsgemeinschaft, project CRC755/B03. R.L. acknowledges funding from the Swedish Research Council (637-2014-6929).

\section{Open Access}

This article is distributed under the terms of the Creative Commons Attribution 4.0 International License (http:// creativecommons.org/licenses/by/4.0/), which permits unrestricted use, distribution, and reproduction in any medium, provided you give appropriate credit to the original author(s) and the source, provide a link to the Creative Commons license, and indicate if changes were made.

\section{References}

1. Paizs, B., Suhai, S.: Fragmentation pathways of protonated peptides. Mass Spectrom. Rev. 24, 508-548 (2005)

2. Laskin, J., Futrell, J.: Collisional activation of peptide ions in FT-ICR mass spectrometry. Mass Spectrom. Rev. 22, 158-181 (2003)

3. Bari, S., Hoekstra, R., Schlathölter, T.: Peptide fragmentation by keV ioninduced dissociation. Phys. Chem. Chem. Phys. 12, 3376-3383 (2010)

4. Bari, S., Hoekstra, R., Schlathölter, T.: Fast side-chain losses in keV ioninduced dissociation of protonated peptides. Int. J. Mass. Spectr. 299, 64 $70(2011)$
5. Hoffmann, W.D., Jackson, G.P.: Charge transfer dissociation (CTD) mass spectrometry of peptide cations using kiloelectronvolt helium cations. J. Am. Soc. Mass Spectrom. 25, 1939-1943 (2014)

6. Zubarev, R., Kelleher, N., McLafferty, F.: Electron capture dissociation of multiply charged protein cations. A nonergodic process. J. Am. Chem. Soc. 120, 3265-3266 (1998)

7. Chakraborty, T., Holm, A.I.S., Hvelplund, P., Nielsen, S.B., Poully, J., Worm, E.S., Williams, E.R.: On the survival of peptide cations after electron capture: role of internal hydrogen bonding and microsolvation. J. Am. Soc. Mass Spectrom. 17, 1675-1680 (2006)

8. Brodbelt, J.S.: Photodissociation mass spectrometry: new tools for characterization of biological molecules. Chem. Soc. Rev. 43, 2757-2783 (2014)

9. Reilly, J.P.: Ultraviolet photofragmentation of biomolecular ions. Mass. Spectr. Rev. 28, 425-447 (2009)

10. Bari, S., Gonzalez-Magaña, O., Reitsma, G., Hoekstra, R., Werner, J., Schippers, S., Schlathölter, T.: Dissociation of a free protonated peptide by VUV photons. J. Chem. Phys. 134, 024314 (2011)

11. Milosavljević, A.R., Nicolas, C., Lemaire, J., Dehon, C., Thissen, R., Bizau, J., Refregiers, M., Nahon, L., Giuliani, A.: Photoionization of a protein isolated in vacuo. Phys. Chem. Chem. Phys. 13, 15432-15436 (2011)

12. Zubavichus, Y.; Shaporenko, A.; Grunze, M.; Zharnikov, M. Innershell absorption spectroscopy of amino acids at all relevant absorption edges. 2005, 109, 6998-7000

13. Stewart-Ornstein, J., Hitchcock, A.P., Cruz, D.H., Henklein, P., Overhage, J., Hilpert, K., Hale, J.D., Hancock, R.E.W.: Using intrinsic $\mathrm{X}$-ray absorption spectral differences to identify and map peptides and proteins. J. Phys. Chem. B. 111, 7691-7699 (2007)

14. Zubavichus, Y., Shaporenko, A., Grunze, M., Zharnikov, M.: Is X-ray absorption spectroscopy sensitive to the amino acid composition of functional proteins? J. Phys. Chem. B. 112, 4478-4480 (2008)

15. Gonzalez-Magaña, O., Reitsma, G., Tiemens, M., Boschman, L., Hoekstra, R., Schlathölter, T.: Near edge X-ray absorption mass spectrometry of a gas-phase peptide. J. Phys. Chem. A. 116, 10745 (2012)

16. Milosavljević, A.R., Nicolas, C., Rankovic, M.L.J., Canon, F., Miron, C., Giuliani, A.: K-shell excitation and ionization of a gas-phase protein: interplay between electronic structure and protein folding. J. Phys. Chem. Lett. 6, 3132-3138 (2015)

17. Egorov, D., Hoekstra, R., Schlathölter, T.: A comparative VUV absorption mass-spectroscopy study on protonated peptides of different size. Phys. Chem. Chem. Phys. 19, 20608-20618 (2017)

18. Egorov, D., Schwob, L., Lalande, M., Hoekstra, R., Schlathölter, T.: Near edge X-ray absorption mass spectrometry of gas phase proteins: the influence of protein size. Phys. Chem. Chem. Phys. 18, 26213-26223 (2016)

19. Schlathölter, T., Reitsma, G., Egorov, D., Gonzalez-Magana, O., Bari, S., Boschman, L., Bodewits, E., Schnorr, K., Schmid, G., Schroeter, C.D., Moshammer, R., Hoekstra, R.: Multiple ionization of free ubiquitin molecular ions in extreme ultraviolet free-electron laser pulses. Angewandte Chemie-International Edition. 55, 10741-10745 (2016)

20. Florance, H.V., Stopford, A.P., Kalapothakis, J.M., McCullough, B.J., Bretherick, A., Barran, P.E.: Evidence for a-helices in the gas phase: a case study using Melittin from honey bee venom. Analyst. 136, 3446 (2011)

21. Bari, S., Egorov, D., Jansen, T.L., Boll, R., Hoekstra, R., Techert, S. Zamudio-Bayer, V., Bülow, C., Lindblad, R., Leistner, G., Lawicki, A., Hirsch, K., Miedema, P.S., von Issendorff, B., Lau, J.T., Schlathölter, T.: Soft X-ray spectroscopy as a probe for gas-phase protein structure: electron impact ionization from within. Chem. Eur. J. 0, (2018)

22. Niemeyer, M., Hirsch, K., Zamudio-Bayer, V., Langenberg, A., Vogel, M., Kossick, M., Ebrecht, C., Egashira, K., Terasaki, A., Moeller, T., Issendorff, B.V., Lau, J.T.: Spin coupling and orbital angular momentum quenching in free iron clusters. Phys. Rev. Lett. 108, 057201 (2012)

23. Hirsch, K., Zamudio-Bayer, V., Langenberg, A., Niemeyer, M., Langbehn, B., Moeller, T., Terasaki, A., von Issendorff, B., Lau, J.T.: Magnetic moments of chromium-doped gold clusters: the Anderson impurity model in finite systems. Phys. Rev. Lett. 114, 087202 (2015)

24. Feyer, V., Plekan, O., Richter, R., Coreno, M., Prince, K.C., Carravetta, V.: Core level study of alanine and threonine. J. Phys. Chem. A. 112, 7806-7815 (2008)

25. Milosavljević, A.R., Canon, F., Nicolas, C., Nahon, C.M.L., Giuliani, a.A.: Gas-phase protein inner-shell spectroscopy by coupling an ion trap with a soft X-ray beamline. J. Phys. Chem. Lett. 3, 1191-1196 (2012) 
26. Zhang, W., Carravetta, V., Plekan, O., Feyer, V., Richter, R., Coreno, M., Prince, K.C.: Electronic structure of aromatic amino acids studied by soft X-ray spectroscopy. J. Chem. Phys. 131, 035103 (2009)

27. Plekan, O., Feyer, V., Richter, R., Coreno, M., de Simone, M., Prince, K.C., Carravetta, V.: An X-ray absorption study of glycine, methionine and proline. J. Electron Spectrosc. Relat. Phenom. 155, 47-53 (2007)

28. Evans, S.E., Lueck, N., Marzluff, E.M.: Gas phase hydrogen/deuterium exchange of proteins in an ion trap mass spectrometer. Int. J. Mass. Spectr. 222, 175-187 (2003)

29. Journel, L., Guillemin, R., Haouas, A., Lablanquie, P., Penent, F., Palaudoux, J., Andric, L., Simon, M., Ceolin, D., Kaneyasu, T., Viefhaus, J., Braune, M., Li, W.B., Elkharrat, C., Catoire, F., Houver, J., Dowek, D.: Resonant double Auger decay in carbon K-shell excitation of CO. Phys. Rev. A. 77, 042710 (2008)

30. Brook, E., Harrison, M., Smith, A.: Measurements of electron-impact ionization cross-sections of $\mathrm{He}, \mathrm{C}, \mathrm{O}$ and $\mathrm{N}$ atoms. J. Phys B-Atom Mol. Opt. Phys. 11, 3115-3132 (1978)

31. Kim, Y., Desclaux, J.: Ionization of carbon, nitrogen, and oxygen by electron impact. Phys. Rev. A. 66, 012708 (2002)

32. Blanco, F., Garcia, G.: Screening corrections for calculation of electron scattering differential cross sections from polyatomic molecules. Phys. Lett. A. 330, 230-237 (2004)

33. Blanco, F., García, G.: Screening corrections for calculation of electron scattering from polyatomic molecules. Phys. Lett. A. 317, 458-462 (2003)

34. Sanchez-Gonzalez, A., Barillot, T.R., Squibb, R.J., Kolorenc, P., Agaker, M., Averbukh, V., Bearpark, M.J., Bostedt, C., Bozek, J.D., Bruce, S. Montero, S.C., Coffee, R.N., Cooper, B., Cryan, J.P., Dong, M., Eland, J.H.D., Fang, L., Fukuzawa, H., Guehr, M., Ilchen, M., Johnsson, A.S., Liekhus-S, C., Marinelli, A., Maxwell, T., Motomura, K., Mucke, M. Natan, A., Osipov, T., Ostlin, C., Pernpointner, M., Petrovic, V.S., Robb, M.A., Sathe, C., Simpson, E.R., Underwood, J.G., Vacher, M., Walke, D.J., Wolf, T.J.A., Zhaunerchyk, V., Rubensson, J., Berrah, N., Bucksbaum, P.H., Ueda, K., Feifel, R., Frasinski, L.J., Marangos, J.P.:
Auger electron and photoabsorption spectra of glycine in the vicinity of the oxygen K-edge measured with an X-FEL. J. Phys. B-Atom. Mol. Opt. Phys. 48, 234004 (2015)

35. Mouls, L., Subra, G., Aubagnac, J., Martinez, J., Enjalbal, C.: Tandem mass spectrometry of amidated peptides. J. Mass Spectrom. 41, 14701483 (2006)

36. Canon, F., Milosavljević, A.R., Nahon, L., Giuliani, A.: Action spectroscopy of a protonated peptide in the ultraviolet range. Phys. Chem. Chem. Phys. 17, 25725-25733 (2015)

37. Laskin, J., Yang, Z., Ng, C.M.D., Chu, I.K.: Fragmentation of alpharadical cations of arginine-containing peptides. J. Am. Soc. Mass Spectrom. 21, 511-521 (2010)

38. Xia, Q., Lee, M.V., Rose, C.M., Marsh, A.J., Hubler, S.L., Wenger, C.D., Coon, J.J.: Characterization and diagnostic value of amino acid side chain neutral losses following electron-transfer dissociation. J. Am. Soc. Mass Spectrom. 22, 255-264 (2011)

39. Kjeldsen, F., Savitski, M.M., Adams, C.M., Zubarev, R.A.: Determination of the location of positive charges in gas-phase polypeptide polycations by tandem mass spectrometry. Int. J. Mass Spectrom. 252, 204-212 (2006)

40. Morrison, L.J., Brodbelt, J.S.: Charge site assignment in native proteins by ultraviolet photodissociation (UVPD) mass spectrometry. Analyst. 141, 166-176 (2016)

41. Canon, F., Milosavljević, A.R., van der Rest, G., Refregiers, M., Nahon, L., Sarni-Manchado, P., Cheynier, V., Giuliani, A.: Photodissociation and dissociative photoionization mass spectrometry of proteins and noncovalent protein-ligand complexes. Angew. Chem-Int Ed. 52, 83778381 (2013)

42. Harrison, A., Young, A.: Fragmentation reactions of deprotonated peptides containing proline. The proline effect. J. Mass Spectrom. 40, 1173$1186(2005)$

43. Smith, R., Loo, J., Loo, R., Busman, M., Udseth, H.: Principles and practice of electrospray ionization - mass-spectrometry for large polypeptides and proteins. Mass Spectrom. Rev. 10, 359-451 (1991) 